\title{
Metapragmatic explicitation and social attribution in Social Communication Disorder and Developmental Language Disorder: a comparative study.
} DOI:

10.1044/2017_JSLHR-L-17-0026

\section{Document Version}

Accepted author manuscript

Link to publication record in Manchester Research Explorer

Citation for published version (APA):

Adams, C., Lockton, E., \& Collins, A. (2018). Metapragmatic explicitation and social attribution in Social Communication Disorder and Developmental Language Disorder: a comparative study. Journal of Speech, Language, and Hearing Research ,61. https://doi.org/10.1044/2017_JSLHR-L-17-0026

Published in:

Journal of Speech, Language, and Hearing Research

\section{Citing this paper}

Please note that where the full-text provided on Manchester Research Explorer is the Author Accepted Manuscript or Proof version this may differ from the final Published version. If citing, it is advised that you check and use the publisher's definitive version.

\section{General rights}

Copyright and moral rights for the publications made accessible in the Research Explorer are retained by the authors and/or other copyright owners and it is a condition of accessing publications that users recognise and abide by the legal requirements associated with these rights.

\section{Takedown policy}

If you believe that this document breaches copyright please refer to the University of Manchester's Takedown Procedures [http://man.ac.uk/04Y6Bo] or contact uml.scholarlycommunications@manchester.ac.uk providing relevant details, so we can investigate your claim.

\section{OPEN ACCESS}


REVISED VERSION 10 October 2017

Title page

Metapragmatic explicitation and social attribution in Social Communication Disorder and Developmental Language Disorder: a comparative study.

Authors:

Catherine Adams, Elaine Lockton and Anna Collins

Address for correspondence:

Dr Catherine Adams

School of Health Sciences

Ellen Wilkinson Building

University of Manchester

Oxford Road

Manchester

M13 9PL

UK

Tel: +44 1612753368

Email: catherine.adams@manchester.ac.uk 


\section{Abstract}

Purpose: To investigate metapragmatic ability in 6-11 year old children with social communication disorder (SCD), developmental language disorder (DLD) and typical language development (TLD); to explore factors associated with metapragmatic explicitation and social understanding.

Method: In this cross-sectional study, all participants $(\mathrm{N}=82)$ completed an experimental task, the Assessment of Metapragmatics (AMP), in which pragmatic errors are identified in filmed interactions. Responses were scored for complexity/type of explicitation (MP score) and attribution of social characteristics to the films' characters (Social Understanding (SU) score).

Results: SCD and DLD groups had significantly lower MP scores and less sophisticated explicitation than the TLD group. After controlling for language and age, the SCD group had significantly lower SU scores than the DLD group. Significant correlations were found between MP scores and age/language ability but not with pragmatic impairment.

Conclusions: Children with SCD or DLD performed poorly on a metapragmatic task compared to typically developing children, but do not differ from each other in ability to reflect verbally on pragmatic features in interactions. Metapragmatic ability appears to be closely related to structural language ability. The limited ability of children with SCD to attribute social/psychological states to interlocutors may indicate additional social attribution limitations. 


\section{Introduction}

Theoretical accounts of pragmatics have evolved from semiotic or code models in which pragmatic 'signs' are decoded, to contemporary inferential or cognitive accounts in which interlocutors create hypotheses about speakers' intentions (Wilson \& Sperber, 2015). In clinical pragmatics, as applied to developmental communication disorders, there has been a parallel but slower evolution.

Observational and elicitation-based assessments of pragmatics (e.g., Gilliam \& Miller, 2006; Landa, 2013; Phelps-Terasaki \& Phelps Gunn, 2007; O’Neill, 2007) are being supplemented by procedures which address what the child can process about pragmatic information in social interactions (e.g., Bowers, Huisingh \& LoGiudice, 2008). Therapeutic approaches which aim to support training of verbally-mediated social cognitive skills (Timler, Olswang \& Coggins, 2005) are increasingly understood as important. However, there is relatively little research that clarifies the nature of pragmatic interpretation of communication interactions in children with communication disorders. This information has the potential to support potential mechanisms of pragmatic intervention.

In this paper, we investigate metapragmatics as one aspect of pragmatic processing in children with Social Communication Disorder (SCD) or Developmental Language Disorder (DLD) ${ }^{1}$. We define metapragmatics as the child's explicit knowledge of pragmatics in social interactions. The choice of metapragmatics as the focus of this study was motivated by the importance of meta- level interventions in clinical therapeutic practice. Meta-level therapies are abundant in speech-language

\footnotetext{
${ }^{1}$ The term Developmental Language Disorder will be used in preference to Specific Language Impairment, in line with the recommendations made in the CATALISE study (Bishop, Snowling, Thompson, Greenhalgh and the CATALISE consortium 2016).
} 
pathology and meta-level training remains one of the reliable methodologies in the speech-pathologist's toolkit (Law, Campbell, Roulstone, Adams \& Boyle 2008; ASHA Practice Portal n.d.) This is the case across the domains of phonology (Gillon \& Dodd, 1995; Van Kleeck, Gillam \& McFadden, 1998), syntax and morphology (Ebbels, 2007; Zwitserlood, Wijnen, Weerdenburg \& Verhoeven, 2015) and semantics (Bryan, 1997). In contrast, research in clinical metapragmatics is sparse, despite recommendations for its use in therapy for children with pragmatic language difficulties and autism spectrum disorders (Adams, Lloyd, Aldred \& Baxendale, 2006; Adams \& Gaile, 2015; Brinton \& Fujiki, 2006). There are a number of broader pragmatic language therapy studies for children who have autism or language disorder with treatment protocols that include metapragmatic content, e.g., Adams (2006), Adams, Gaile, Earl, Lockton \& Freed (2012), Ingersoll, Dvortcsak, Whalen \& Sikora (2005), Merrison \& Merrison (2005) and Richardson \& Klecan-Aker (2000). The description of metapragmatic content varies in these studies considerably from therapy tasks written as metapragmatic activities (Adams et al 2012) to implied metapragmatic content, such as identification of conversational repair (Merrison \& Merrison 2005).

\section{Metapragmatics}

The term metapragmatic ability (MPA) will be used to describe explicit reflection on the use of pragmatics in verbal interaction. MPA research with children who have typical language development (TLD) consists of studies on individual aspects of pragmatics, such as non-literal language forms (Bernicot, Laval \& Chaminaud, 2007; Laval, 2003), request politeness (Axia \& Baroni, 1985; Baroni \& Axia, 1989; Tsuji and Doherty, 2014) and request appropriacy (Wilkinson \& Milosky, 1987; Bernicot, 
1991). In typical language development, MPA emerges in response to experience of pragmatic behaviours and heard metapragmatic comments. Children are often given instructions containing explicit metapragmatic information (e.g., "remember to take turns", "please don't interrupt") and are expected to decode these statements in order to successfully communicate and interact (Yifat \& Zadunaisky-Ehrlich, 2008). Children between two and four years of age use a variety of metapragmatic behaviours including comments to regulate and maintain the conversation (Becker, 1988). Children under the age of five are able to complete simple experimental metapragmatic tasks (Skarakis-Doyle, 2002) but the development of MPA continues well into adolescence (Wilkinson \& Milosky, 1987).

Studies have concluded that MPA development is associated with the cognitive ability to represent a situation from different interlocutor perspectives and to link linguistic and contextual information (Bernicot, 1991; Bernicot et al., 2007; Laval, 2003; Schwe \& Markman, 1997; Wilkinson \& Milosky, 1987). During development, the child becomes more skilful in explicitly reflecting on the pragmatic 'rules' and rule violations using a range of information. This information includes attribution of the speaker's social intentions and the interlocutor's desires (Hickman et al., 1993; Laval, 2003). Thus metapragmatic ability goes beyond awareness of language form in that knowledge must also be invoked about the social and physical context of interactions and the motivation of participants. Given this evidence, an assessment of MPA ought to integrate task questions that require social attribution of interlocutors' characteristics and intentions. 
Explicitation is defined as a process of verbal reflection in which metapragmatic judgements have undergone a process of "representational redescription" (Karmiloff-Smith, 1986) in which the knowledge or procedure that was involved in processing becomes available to conscious thought. Baroni \& Axia (1989) adapted Karmiloff-Smith's theoretical model of metalinguistic awareness in order to describe the emergence of four levels of sophistication in children's awareness of pragmatics. The first level is implicit knowledge or non-awareness in which the child cannot identify or talk about the pragmatic behaviour or rule. This is then followed by a stage in which there is some metalinguistic awareness of the event but the pragmatic behaviour or rule cannot be explicitly talked about. The two further stages of explicitation of pragmatics are defined as 'linguistic marker awareness', in which the aspect of the language marking the pragmatic behaviour can be explicitly reflected upon; and finally, the stage of 'pragmatic rule awareness', in which the child is able to explicitly reflect on the pragmatic rule e.g., "he was impolite". Partial validation of this sequence was provided by Collins, Lockton \& Adams (2014) who used this framework to show that there was a linear relationship between age and explicitation ability in typically developing children aged between six and ten years.

\section{Pragmatics in Social Communication Disorder}

Children who have persistent difficulty with the acquisition of both pragmatics and language have been described as having a Social Communication Disorder (DSM-V, American Psychiatric Association, 2013) or pragmatic language impairment (Bishop, 2000). SCD is distinguished from Developmental Language Disorder in that pragmatics in children with SCD is weak compared to relative strength in structural aspects of language such as syntax and morphology (Bishop, 200). DLD is typically 
characterised as a persistent disorder of language form and content (Tomblin et al., 1997) that is unexplained by any obvious developmental cause.

The main observable pragmatic characteristics of children with SCD include difficulty with presupposition and reference; tendency to hold the conversational floor; poor cohesion and coherence in discourse; difficulty in signalling topic shift and change; and a difficulty in interpreting and meshing subtle verbal and non-verbal clues to the speaker's intent (Adams, 2013; Bishop, 2000). The nature of the observed pragmatic impairment in SCD is variable but has, in some individuals, similarities to those observed in high functioning autism (Tager-Flusberg, Paul \& Lord, 2005) such as unusual use of volume/pitch and nonverbal aspects of communication such as eye contact ( Bishop, Chan, Adams, Hartley \& Weir, 2000). Children with SCD may have difficulties with social interaction but do not meet diagnostic criteria for autism. Gibson, Adams, Lockton and Green (2013) found that both an SCD group and a DLD group had social interaction difficulties based on a systematic observation of playground peer interactions. Children with SCD had more social difficulties than children with DLD but less than a group with high functioning autism. Many children with SCD also have language learning difficulties that are similar although less severe than those seen in DLD. Pragmatic impairments have been found in a proportion of children with DLD (Marton, Abramoff \& Rosenzweig, 2005; Meline \& Brackin, 1987) but these are generally considered to be associated with poverty of expressive language skills and delayed comprehension development.

There is an assumption that children with SCD are likely to have very limited MPA given their persistent, observable pragmatic difficulties in discourse and 
conversation, but this has not been examined in comparison to children with TLD or DLD. It may be that variance in metapragmatic ability can be fully explained by current pragmatic difficulties, or it could be that MPA is related to structural language skills such as sentence comprehension and construction. If the latter is the case, we would expect there to be no difference between DLD and SCD groups on metapragmatic explication when language levels are controlled. We would predict that both groups would show limited MPA compared to children with typical language development since they both have developmental communication limitations. MPA may also be related to developmental social interaction difficulties. If this is the case we would expect children with SCD to have more limited metapragmatic explicitation ability and difficulty with social attribution to interlocutors compared to children with DLD.

\section{Aims}

This study asks whether children with SCD perform differently on a novel metapragmatic task to children with typical language development (children with TLD) and children with DLD, both in terms of overall ability and of levels of explicitation shown; and to what extent factors such as language ability, reported pragmatic ability and history of social interaction difficulties account for performance on that task. The study will also explore children's social attribution to participants in communicative exchanges during the MP task with the aim of establishing if there is a difference between children with SCD and children with DLD in their knowledge about speakers' potential social intentions. This study addressed five research questions. 
1) Do children with SCD perform differently on a metapragmatic task to children with typical language development (children with TLD) and children with DLD? If MPA is linked to pragmatic ability then metapragmatic task performance (Total MP Score) of children with SCD will be poorer than performance of children with TLD and poorer than performance of children with DLD when structural language ability is controlled for.

2) Do children with SCD perform differently in their knowledge about speakers' potential social intentions of participants in communicative exchanges during the MP task (Social Understanding score)? We predict that there will be differences between the groups; TLD children will score more highly on SU than both SCD and DLD. If SU is linked to the purported social difficulties of children with SCD, then SU scores will be poorer than performance of children with DLD when structural language ability is controlled for.

3) Do children with SCD perform differently on levels of explicitation observed in the metapragmatic task to children with typical language development (children with TLD) and children with DLD? We predict that children with SCD and DLD will produce simpler levels of explicitation on the MP task compared to children with TLD, since their language abilities are by definition lower. No predictions are made about differences between SCD and DLD groups and this analysis will be exploratory.

4) Is there any difference in explicitation of responses to the Social Understanding part of the MP task between the TLD, SCD and DLD groups? We predict that children with SCD and DLD will produce simpler, less sophisticated examples of social understanding on this task compared to 
children with TLD, since their language abilities are by definition poorer. If SU is linked to the purported social difficulties of children with SCD, then they may perform more poorly on level of SU explicitation than children with DLD.

5) What are the predictors of MP performance for children with SCD? Contribution of age, non-verbal reasoning, language ability and observable pragmatic behaviours in the SCD will be explored to assess correlations with MP and SU scores.

\section{Method}

\section{Participants}

Thirty-four children with SCD aged 6;1-10;9 and fourteen children with DLD aged 6;2-10;10 were recruited from UK NHS speech and language therapists; all were attending mainstream elementary schools and were identified as having Special Educational Needs. None of the children in this study had a current diagnosis of autism. As a precursor to recruitment, local therapists were asked to refer children whom they considered to have pragmatic language difficulties using a Social Communication Behavior Checklist designed for a parallel study (SCBC, see appendix). Children had to display a minimum of two out of five pragmatic behaviours on this list to be considered for inclusion in the SCD group. Local therapists were also asked to refer children they considered to have DLD who would not meet the criteria of $2 / 5$ pragmatic items on the SCBC. After this triage procedure, assessment of Social Communication Disorder was made by the specialist 
speech and language therapist (Collins) using the DSM-V description and Norbury's (2014) description of Social (Pragmatic) Language Disorder for guidance:

- disproportionate difficulty in the development of pragmatics (using spoken language for conversation and social interaction)

- condition that has existed from early childhood

- absence of autism diagnosis

- subtle difficulties with high-level language processing such as making inferences

Assessment of pragmatic function was carried out using the Children's Communication Checklist -2 (CCC-2) (Bishop 2003a). The CCC-2 is a report instrument in which the parent rates his/her child's communication and social behaviour characteristics on a scale of 0-3 in terms of frequency of occurrence. The CCC-2 contains 10 subscales: speech, syntax, semantics, coherence, inappropriate initiation, stereotyped language, use of context, nonverbal communication, social relations and interests. An overall communication 'score' or General Communication Composite (GCC) can be derived which is based on the sum of all scales (except social relations and interests scales). Children who have a GCC of less than 58 are considered to present with a "clinically significant communication problem" (Bishop 2003a, p11). A second index, the Social Interaction Deviance Composite (SIDC), can be derived from the CCC-2 report and can be used to identify children who have disproportionate pragmatic difficulties; that is, their reported pragmatic difficulties are out of proportion to their structural language problems (syntax, semantics). The SIDC is derived by a comparison of the mismatch between 
the sum of scores on the scales: speech, syntax, semantics, coherence with the sum of the scores on the scales: inappropriate initiation, nonverbal communication, social relations, interests. This index is considered to be the best currently available method of systematically distinguishing between different subtypes of language disorder (i.e., SCD and DLD), The third CCC-2 index used in this study was the Pragmatics Composite (CCC-PRAG) which is derived by summing the scales: coherence, inappropriate initiation, stereotyped language use of content, and nonverbal communication. Bishop (2003a) considered the Pragmatics Composite score to be most useful in differentiating children with pragmatic difficulties from children who have typical language development.

Participants recruited to the SCD group all scored in the communication impaired range $(\leq 58)$ on the GCC, and in the range indicative of pragmatic deficit $(\leq 8)$ on the Social Interaction Deviance Composite (SIDC), of the CCC-2 (Bishop, 2003a), a parent report checklist of current functional communication and pragmatics. Children with DLD scored in the communication impairment range $(\leq 58)$ on the GCC, and in the non-pragmatic deficit range $(\geq 9)$ on the SIDC of the CCC-2. Children with DLD also had either a standard score $<8$ on the Naming subtest of the Assessment of Comprehension and Expression 6-11 (ACE) (Adams, Cooke, Crutchley, Hesketh \& Reeves, 2001) and/or a standard score $<80$ on the Test for Reception of Grammar (TROG-2, Bishop, 2003b). There were no inclusion criteria on ACE and TROG for children with SCD as language ability tends to vary more in this group and we were interested to see how language ability related to metapragmatic performance on the experimental task. 
Thirty-four children with TLD aged 6;1-10;10 were recruited via mainstream elementary schools in the North West of England. Children with TLD all achieved a scaled score of $\geq 7$ on the ACE Naming subtest and a standard score $>85$ on the TROG-2. All participants had English as their primary language of communication and learning in school and had no evidence of severe difficulties in emotional development, behavior needs, unintelligibility or hearing impairment as observed by the referrer. All participants scored within the normal range ( $\geq 5$ th centile) on the Raven's Coloured Progressive Matrices (RCPM; Raven, 1979), a test of non-verbal analogical reasoning skills. Participants with TLD were not matched to children in the SCD or DLD groups but between groups comparisons were carried out to check for differences on key characteristics (see results section).

\section{Materials}

In addition to measures used as inclusion criteria, children with SCD also completed the Clinical Evaluation of Language Fundamentals - Fourth Edition UK (CELF-4 UK, Semel, Wiig \& Secord, 2006). Receptive and Expressive composites were calculated and these were used in later analysis of structural language ability. For children with SCD or DLD the Pragmatics Composite (CCC-PRAG) of the CCC-2 was computed, providing an indication of degree of reported pragmatic difficulties. Parents of children with SCD and children with DLD also completed the Social Communication Questionnaire Lifetime version (SCQ, Rutter, Bailey \& Lord, 2003), a parent questionnaire which asks about the history of diagnostic features of autism/social interaction difficulties over the child's lifetime. CCC-2 parent report forms and SCQ questionnaires were completed by a parent of each participant either during the 
language measures assessment session or were sent home and returned by post to the principal researcher's lab.

All children completed the Assessment of Metapragmatics (AMP, Collins et al, 2014), a novel experimental task that measures ability to identify and describe pragmatic errors. The AMP task consists of three practice films followed by thirteen short films depicting a series of naturalistic social interactions between two child actors from a local drama group (of elementary or high school ages). Actors used prepared scripts written using language and content appropriate for school age children. In each interaction, one child actor makes a pragmatic rule violation and the other child actor displays a typical reaction to that violation. Rule violations adopted in the AMP task were based on the typical pragmatic 'errors' observed in children with SCD in data available to the authors from previous work. A list of pragmatic rule violations in each film is given in the Appendix. Total testing time for the thirteen AMP films was approximately thirty minutes. All assessments, including the AMP task, were completed over two testing sessions in a quiet room in the child's own school. Responses were recorded on a digital audio-recording device and transcribed as soon as possible after the assessment session.

Internal reliability of individual AMP films was explored by establishing correlations amongst metapragmatic scores (MP scores) for each film. Good internal reliability was demonstrated; all film MP scores correlated significantly with each other. Correlations ranged from .595 to .669 and all correlations were significant at the 0.01 level (one-tailed) using Spearman's Rho. Analysis using Cronbach's alpha for individual films was 0.915 , indicating a good internal consistency. 
Each AMP film (which contained a single pragmatic 'error') was presented to participants on a laptop computer. After participants had viewed each film twice, the researcher asked the participant: 1) MP questions; a set of three questions designed to assess explicit understanding of the pragmatic error. These were (i) MP question 1 (Descriptive question): "Something went wrong in the conversation. What went wrong?; (ii) MP question 2 (Reflective question): "Why is that wrong?” (iii) MP question 3 (Rule question): "What could the boy have done differently?" and 2) a Social Understanding (SU) question designed to elicit social understanding or attribution of the person performing the pragmatic 'error' in that film (e.g., "What kind of person is that girl?"). Social understanding in this context was defined as the ability to identify and describe a social, psychological or emotional state associated with the pragmatic error shown on the film. Questions were asked whilst looking at a pre-determined freeze frame from that film. One repetition of each question was allowed and one prompt could be used to elicit a more detailed response from the child (e.g., "tell me more" / "anything else?").

Participants' responses to MP and SU questions were transcribed verbatim and scored according to the level of verbal explicitation demonstrated using a scoring guide (Collins et al, 2014). Responses were awarded zero points for non-awareness of pragmatics or for no response, one point for demonstrating linguistic marker awareness (Baroni \& Axia's secondary explicitation), or two points for demonstrating the more sophisticated pragmatic rule awareness (Baroni \& Axia's tertiary explicitation). Each response to the MP Questions (Descriptive, Reflective and Rule) was scored using this scheme. Scores for the three MP questions were then summed for each film. A total metapragmatic score (Total MP score) was derived by summing 
scores for the 13 films. A separate scoring scheme was developed to differentiate levels of sophistication in responses to the Social Understanding Question. This scoring scheme was exploratory and was broadly based on the levels of sophistication outlined in the Person Index Score of Klin's Social Attribution Task (Klin, 2000). Three levels of sophistication were applied: zero points were awarded for non-awareness or no response, one point was awarded for describing the pragmatic behaviour, and two points were awarded for attributing a social, psychological or emotional state. Examples of the scoring guides are shown in the Appendix.

Reliability of transcription (by the third author) was checked by a graduate psychologist, experienced in transcribing child language, who retranscribed six complete sets of test data. Recordings from two children from each group were retranscribed making a total of 312 responses that were subject to checks. Agreement on transcription was made when both transcribed versions of a response (MP and SU question responses) was substantially the same in terms of number of words, sentence structure, vocabulary and any notes regarding unintelligible speech, to the extent that the initial transcription and retranscription would be likely to be coded in the same manner. Treating paired ratings for transcribed responses as the sampling unit, Cohen's kappa was 0.926, indicating excellent agreement.

For inter-rater reliability of MP and SU coding using the scoring guide, 412 responses (8 transcripts) of three children from SCD and TLD groups and two children from the DLD group (not including the children included in the retranscription analysis) were 
subject to checks between the third author and a second graduate psychologist. Substantial inter-rater agreement was found (Cohen's kappa $=0.783$ ).

\section{Results}

Plan of analysis

Analysis commenced with a between groups comparison of group characteristics to check for differences on age and RCPM, using ANOVA with correction for multiple comparisons. Language status of the two clinical groups was further compared using non-parametric statistics since group sizes were uneven. Analyses then considered the main between groups comparisons on Total MP Score and Total SU Score (Research questions 1 and 2) using ANCOVA. It was hypothesised that the TLD group would score more highly than SCD and DLD on both measures, but there was no strong prediction for differences between SCD and DLD since both groups have significant but variable language difficulties that may contribute to limitations in metapragamtic ability and social understanding. Variability in age and non-verbal reasoning were controlled in this analysis by using age (in months) and RPCM as covariates; these were chosen as they were found to be significantly related to MP scores in previous analysis of the same dataset (Collins et al, 2014) and because the DLD group showed a non-significant trend to have lower RPCM scores than the SCD group. A correlation analysis of age, RCPM, language test scores and Total MP/SU total scores is provided in Table 1 showing positive correlations between Total MP scores, Total SU scores and language test scores (except the ACE Naming subtest). Given that the ability to use language to express ideas is a necessary feature of explicit MPA, there was a strong rationale for exploring the contribution that structural language ability makes to MPA. The language measure used for all three 
groups (TROG-2) is included as a covariate where all groups are included in the ANCOVA. CELF-4 UK Receptive Language Index (CELF-RLI) and CELF-4 UK Expressive Language Index (CELF-ELI) are included as covariates in further analysis of the relationship between language ability and MP ability for the clinical groups only, since TLD children did not complete the CELF test. All tests are two-tailed except where explicitly stated.

Children's ability to explain their metapragmatic knowledge (Total MP Explicitation Score) and social understanding choices (Total SU Explicitation score) were further explored using ANCOVA to control for age and non-verbal reasoning (Research questions 3 and 4). Between groups analysis of SCD and DLD on MP scores was exploratory since both groups have significant language difficulties that may contribute to limitations in explicitation. However it was expected that SCD may have lower scores than DLD on SU explicitation scores due to their known limitations in social understanding.

Since this study was principally concerned with a possible metapragmatic deficit in the SCD group, potential predictors (Research question 5) of MP and SU were further explored for that group only, using correlations between the dependent variables Total MP score and Total SU score with age, RCPM and language measures (TROG-2, CELF RLI, CELF ELI scores) and pragmatic ability (CCC-PRAG). This analysis was exploratory only. Correlations between Total MP score, language ability and age would be expected; for Total SU score similar predictors would hold but there may be additional prediction from observed pragmatic limitations as measure on the CCC-PRAG. 
Table 1 about here

\section{Group characteristics}

The sample characteristics for the TLD, SCD and DLD groups are shown in Table 2. Differences in mean scores between groups were explored using ANOVA Welch $F$ and post-hoc tests, using Games-Howell correction for multiple comparisons. Group differences on age $(F(2,34)=1.51, p>0.05)$ and Raven's $\operatorname{CPM}(F(2,42)=2.79, p>0.05)$ were found to be non-significant, however, there was a trend for children with DLD to have lower Raven's CPM scores. Comparison of group means revealed significant group differences on both TROG-2 $(F(2,32)=55.41, p<.001)$ and ACE Naming $(F(2$, $49)=38.83, p<.001)$. Post-hoc analysis showed the TLD group to have significantly higher scores than DLD and SCD groups on both measures, while these latter groups did not significantly differ from each other.

Differences in mean scores between SCD and DLD groups, on additional measures of language, pragmatics and history of autistic features used with these groups only, were explored using the Mann-Whitney test. Differences between SCD and DLD groups on CELF-Receptive Language Index (RLI) were found to be non-significant ( $U$ = $167, \mathrm{~ns})$. SCD and DLD groups were significantly different on CELF-Core Language Score $(C L S)(U=109.5, p<.005)$ and CELF- Expressive Language Index $(E L I),(U=89.5$, $p<.001$ ), indicating better structural language skills in the SCD group. The SCD group had significantly lower scores than the DLD group on the CCC-PRAG ( $U=123$, $\mathrm{p}<.005)$ indicating greater pragmatic impairment in the former group as expected. Higher scores on the SCQ $(U=97.5, p<.005)$ for the SCD group as compared to DLD 
indicated a higher frequency of autistic features in the child's developmental history as rated by a parent or caregiver.

Differences in mean scores between SCD and DLD groups, on additional measures of language, pragmatics and history of autistic features used with these groups only, were explored using the Mann-Whitney test. Differences between SCD and DLD groups on CELF-RLI $(U=167, p>0.05)$ were found to be non-significant. SCD and DLD groups were significantly different on CELF- ELI, $(U=89.5, p<.001)$, indicating better expressive ability in the SCD group. The SCD group had significantly lower scores than the DLD group on the CCC-PRAG $(U=123, p<.005)$ indicating greater pragmatic impairment in the former group as expected. Higher scores on the SCQ $(U=97.5, p<.005)$ for the SCD group as compared to DLD indicated a higher frequency of autistic features in the child's developmental history as rated by a parent or caregiver.

A gender analysis was carried out using the whole sample as one group, since female numbers were low in the SCD group and SLI sample size was small (male to female participant numbers are shown in Table 2). There was a preponderance of male participants in the sample overall: 57 makes and 25 females. Analysis of variance was used to compare male and females on inclusion criteria. . No significant differences between sexes was found in the inclusion measures: Age $(1,45)$ F1.186 ns; RPCM F $(1,12)$ ns.; TROG-2 $F(1,27)$ ns.; ACE Naming F $(1,13)=1.258$, ns.

Table 2 here 
Thirteen participants across the three groups had missing MP responses due to unintelligible or inaudible recordings. No one participant had more than four out of a total of thirty-nine possible responses missing. Missing values were replaced with the average of the non-missing values for that specific question from the same participant's data.

Table 3 here

Means, standard deviations and ranges for MP scores for TLD, SCD and DLD groups are presented in Table 3. Analysis of Covariance (ANCOVA) was used to investigate whether mean Total MP scores differed between the TLD, SCD and DLD groups. Age and RCPM were chosen as covariates for the reasons stated above. Planned contrasts (simple) were used with SCD as reference category. The covariate, age, was significantly related to Total MP score $(F(1,77)=29.4, p<0.001)$. There was no significant effect of RCPM on Total MP score after controlling for the effect of age $(F(1,77)=2.08, n s)$. There was a significant effect of group on Total MP score after controlling for age and $\operatorname{RCPM}(F(2,77)=34.47, \mathrm{p}<0.001)$. Planned contrasts revealed that the SCD group had significantly lower Total MP score compared to the TLD group $(t(77)=7.19, p<0.001)$ but not significantly different Total MP score to the DLD group $(t(77)=-1.47, n s)$. The estimated group differences $(95 \% \mathrm{Cl})$ were 19.22 (13.9 to 24.54$)$ and $-5.1(-12.03$ to 1.82$)$ respectively.

ANCOVA was further used to investigate whether mean Total MP scores differed between the SCD and DLD groups only, after controlling for structural language measures. Age, TROG-2, CELF-ELI and CELF-RLI were used as covariates. Age, was significantly related to Total MP score $(F(1,41)=10.41, p<0.005)$. There was no 
significant effect of TROG-2 $(F(1,41)=.899, n s)$ and CELF-RLI $(F(1,41)=.052$, ns $)$ on Total MP score after controlling for the effect of age. The covariate, CELF-ELI, was significantly related to Total MP score $(F(1,41)=6.08, p<0.05)$. There was no significant effect of group on Total MP score after controlling for age and structural language measures $(F(1,41)=.295, \mathrm{~ns})$.

Between groups comparison on total social understanding score (Research question 2)

Means and standard deviations for Total SU scores for TLD, SCD and DLD groups are presented in Table 4. ANCOVA was used to investigate whether mean Total SU scores differed between the TLD, SCD and DLD groups. Age and RCPM were chosen as covariates. Planned contrasts (simple) were used with SCD as reference category. The covariate, age, was significantly related to Total SU score $(F(1,77)=26.8$, $p<0.001)$. There was also a significant effect of RCPM on Total SU score after controlling for the effect of age $(F(1,77)=6.33, p<.05)$. Further, there was a significant effect of group on Total SU score after controlling for age and RCPM $(F(2,77)=12.79, p<0.001)$. Planned contrasts revealed that the SCD group had significantly lower Total SU scores compared to the TLD group $(t(77)=5.02, p<0.001)$ but not significantly different Total SU score to the DLD group $(t(77)=1.16, n s)$. The estimated group differences $(95 \% \mathrm{Cl})$ were 6.271 (3.785 to 8.756$)$ and $1.886(-1.348$ to 5.12 ) respectively.

Table 4 about here

ANCOVA was further used to investigate whether mean Total SU scores differed between the SCD and DLD groups after controlling for structural language measures. 
Age, RCPM, TROG-2, CELF-ELI and CELF-RLI were used as covariates. The covariate, age, was significantly related to Total SU score $(F(1,40)=22.78, p<0.001)$. There was no significant effect of RCPM $(F(1,40)=.023, n s)$, TROG-2 $(F(1,40)=1.98, n s)$, CELF$\operatorname{RLI}(F(1,40)=1.95, n s)$ or CELF-ELI $(F(1,40)=3.54, n s)$ on Total SU score after controlling for the effect of age. There was a significant effect of group on Total SU score after controlling for age and structural language measures $(F(1,40)=8.12$, $p<0.01)$ with the SCD group scoring significantly lower than the DLD group $(t(40)=$ $2.85, \mathrm{p}<0.01)$. The estimated group difference $(95 \% \mathrm{Cl})$ was 4.72 (1.37 to 8.06).

Between groups comparison on metapragmatic explicitation score (Research question 3)

The mean number of MP responses categorised as non-awareness, linguistic marker and pragmatic rule awareness response types for TLD, SCD and DLD groups are shown in Table 5. Some children in both clinical groups were able to provide some responses demonstrating the most sophisticated level of MP explicitation, pragmatic rule awareness. Separate ANCOVAs were used to investigate whether frequency of awareness/non-awareness responses and frequency of the most sophisticated, pragmatic rule awareness, responses differed between the TLD, SCD and DLD groups. Age was used as a covariate as it was found to be significantly related to Total MP score. Planned contrasts (simple) were used with SCD as reference category. The covariate, age, was significantly related to frequency of nonawareness responses $(F(1,78)=18.16, p<0.001)$. There was a significant effect of group on frequency of non-awareness responses after controlling for age $(F(2,78)=$ $25.05, p<0.001)$. Planned contrasts revealed that the SCD group were significantly 
more likely to provide non-awareness responses than the TLD group $(t / 78)=-5.72$, $p<0.001$ ) but frequency of non-awareness responses in the SCD group did not significantly differ to the DLD group $(t(78)=1.76, n s)$. The estimated group differences $(95 \% \mathrm{Cl})$ were 4.18 (2.73 to 5.64$)$ and 1.71 (-.23 to 3.65$)$ respectively. The covariate, age, was significantly related to frequency of pragmatic rule awareness responses $(F(1,78)=15.63, p<0.001)$. There was a significant effect of group on frequency of pragmatic rule awareness responses after controlling for age $(F(2,78)=$ $24.96, p<0.001)$. Planned contrasts revealed that the SCD group were significantly less likely to provide pragmatic rule awareness responses compared to the TLD group $(t(78)=5.56, p<0.001)$ and significantly more likely to provide pragmatic rule awareness responses compared to the DLD group $(t(78)=-2, p=.049)$. The estimated group differences $(95 \% \mathrm{Cl})$ were 3.26 (2.09 to 4.43$)$ and 1.56 (.01 to 3.12$)$ respectively.

Table 5 about here

Between groups comparison on social understanding explicitation score (Research question 4)

Table 6 shows the percentage of SU responses categorised as non-awareness, pragmatic behaviour and psychological state awareness for TLD, SCD and DLD groups.

Table 6 here

Separate ANCOVAs were used to investigate whether frequency of awareness/nonawareness responses and frequency of the most sophisticated, psychological state awareness responses differed between the TLD, SCD and DLD groups. Age and RCPM 
were used as covariates as they were found to be significantly related to Total SU score. Planned contrasts (simple) were used with SCD as reference category. The covariate, age, was significantly related to frequency of non-awareness responses $(F(1,77)=24.35, p<0.001)$. There was no significant effect of RCPM on frequency of non-awareness responses after controlling for the effect of age $(F(1,77)=3.34, n s)$. There was a significant effect of group on frequency of non-awareness responses after controlling for age and $\operatorname{RCPM}(F(2,77)=13.3, p<0.001)$. Planned contrasts revealed that the SCD group were significantly more likely to provide non-awareness responses than the TLD group $(t(77)=-5.11, p<0.001)$ but frequency of nonawareness responses in the SCD group did not significantly differ to the DLD group $(t(77)=-1.06, n s)$. The estimated group differences $(95 \% \mathrm{Cl})$ were $-4.79(-6.65$ to $2.92)$ and $-1.29(-3.72$ to 1.14$)$ respectively. The covariates, age $(F(1,77)=10.49$, $p<0.005)$ and $\operatorname{RCPM}(F(1,77)=6.35, p<0.05)$ were significantly related to frequency of psychological state awareness responses. There was a significant effect of group on frequency of psychological state awareness responses after controlling for age and Raven's CPM $(F(2,77)=3.42, p<0.05)$. Planned contrasts revealed that the SCD group were significantly less likely to provide psychological state awareness responses than the TLD group $(t(77)=2.62, p<0.05)$ but frequency of psychological state awareness responses in the SCD group did not significantly differ to the DLD group $(\mathrm{t}(77)=.812, \mathrm{~ns})$. The estimated group differences $(95 \% \mathrm{Cl})$ were $1.48(.35$ to $2.61)$ and .6 (-.87 to 2.07$)$ respectively.

Possible predictors of MP and SU performance for children with SCD (Research question 5) 
Correlational analyses were undertaken to identify potential predictors of MPA for the SCD group only. Spearman's correlations (one-tailed) revealed that there were significant positive correlations between Total MP score and age $(r=.414, p<0.01)$, CELF-RLI $(r=.360, p<0.05)$, CELF-ELI $(r=.572, p<0.001)$, TROG-2 $(r=.515, p<0.005)$, and ACE Naming $(r=.474, p<0.005)$. However, no significant correlations were found between Total MP score and RCPM $(r=.021, n s)$, SCQ $(r=-0.077, n s)$ or CCC-PRAG ( $r$ $=.024, \mathrm{~ns})$.

Correlational analyses were also undertaken to identify potential predictors of SU ability for the SCD group only. Spearman's correlations (one-tailed) revealed that there were significant positive correlations between Total SU score and age $(r=.382$, $p<0.05)$, CELF-ELI $(r=.494, p<0.005)$, TROG-2 $(r=.439, p<0.01)$, and ACE Naming $(r=$ $.355, p<0.05)$. However, no significant correlations were found between Total SU score and Raven's CPM ( $r=.046, n s)$, CELF-RLI $(r=.251, p<0.05)$, SCQ $(r=-0.112, n s)$ or CCC-PRAG $(r=.094, \mathrm{~ns})$.

\section{Discussion}

Results revealed that children with SCD and children with DLD performed poorly on a metapragmatics task compared to children with typical language development. This is not unexpected given the significant correlation of MP scores with a measure of structural language. The SCD group had superior expressive language skills and inferior reported pragmatic ability than the DLD group. However, the hypothesis that the SCD group would perform more poorly on a task of MPA than the DLD group, since the former have disproportionate difficulty with pragmatics, was not supported even when structural language abilities were controlled for. The diagnosis 
of Social Communication Disorder conferred nothing unique to the ability to reflect on pragmatic rules in addition to the presence of a developmental language limitation. This is resonant with other research that has attempted to identify unique characteristics of processing in children with SCD (Botting \& Adams, 2005). Metapragmatic ability was not predicted by pragmatic impairment as measured on the CCC-2. Furthermore, no significant correlations were found in this study between MP score and measures of nonverbal ability or history of autistic features in the SCD group. A likely conclusion is that for most children with communication impairment of the two types studied here, structural language ability is the most significant factor in MP performance. This conclusion fits well with studies that have supported limitations in language processing, and not solely social cognitive deficits, to underlie the pragmatic difficulties of children with autism spectrum disorder, e.g., Norbury \& Bishop (2003), Norbury (2004), Gernsbacher \& Propas-Kapit (2012). Given the close relationship of SCD with ASD it is likely that a similar hypothesis could be made about the role of language processing limitations in SCD: that is, children with SCD have difficulty with metapragmatic tasks and explicitation due to language processing difficulties which are of a relatively subtle nature. This would not explain, however, the unique profile of the SCD group on the Social Understanding questions of the AMP.

The explicitation analysis revealed that children with either SCD or DLD were more likely than children with TLD to produce responses to MP questions in the 'no response or non-awarenesss' categories and less likely to show metapragmatic explication at the highest level of sophistication, pragmatic rule awareness. This is a critical finding as it demonstrates that it is not only the expression of metapragmatic 
knowledge (via complex language explanations in the AMP task) that is impaired in these clinical groups but also the recognition of the pragmatic behaviour in interlocutors. The demonstrated lack of awareness of pragmatic behaviors in filmed interactions would have been anticipated in children with SCD but was not entirely expected in children with DLD. It is commensurate with recent research such as Davies, Andres-Roqeta and Norbury (2016) who found weak pragmatic skills in children with DLD. On the other hand, some SCD and DLD individuals were able to provide some sophisticated pragmatic rule awareness responses. Analysis revealed a wide range of abilities in all groups, with high variability across individuals in the SCD and DLD groups.

SCD and DLD groups both performed poorly on the social understanding aspect of the metapragmatic task compared to children with TLD and SCD/DLD groups mean did not differ. This finding suggests that low SU scores may be partially explained in SCD and DLD by poor structural language skills. More sophisticated responses in AMP SU coding relied on use of abstract terms in verbal responses, particularly in describing psychological states (e.g., "she was selfish"). The acquisition and use of abstract vocabulary is known to be almost universally and persistently delayed in DLD (Sheng \& McGregor, 2010). Poor SU scores may therefore to some extent be attributable to limited lexical-semantic knowledge.

Controlling for structural language scores and age as covariates, children with SCD showed poorer ability to attribute social/psychological states to interlocutors compared to children with DLD. Social understanding scores were not significantly correlated with nonverbal ability, pragmatic ability or history of autistic features measures for children with SCD. Therefore, this finding cannot be explained by the 
presence of autism features in children's development (as measured by SCQ in this study). It is consistent with the conceptualisation of SCD as children who have mild social interaction difficulties but who do not meet strict diagnostic criteria for autism (Gibson et al, 2013). The task of social attribution to characters in the AMP films may be tapping into subtle and persistent difficulties in interpreting social cues in children with SCD during development which, in addition to persistent structural language limitations, contribute to restricted metapragmatic performance and observable, persistent pragmatic deficits. Further research should systematically compare the performance of children with SCD and children with high functioning autism to further explore the role of structural language and social cognition in the interpretation of pragmatic rules.

The study had significant limitations since it relied on a novel assessment procedure that has not been subjected to concurrent validity testing, has been subject to reliability testing only within the research team and test-retest reliability on AMP has not been carried out. Furthermore, the surprising lack of a distinct 'pragmatic difficulty' for the SCD group on the AMP task (compared to the DLD group) should be treated with caution. It could be that the task itself is simply functioning as a highdemand language assessment, and does not tap into the true nature of social cognitive and language problems of SCD. Another limitation is that whereas no children had a diagnosis of autism in the SCD group, we did not carry out assessments for autism ourselves due to time restraints. The diagnosis of SCD remains contentious (Norbury 2014) and the potential overlap into autism spectrum disorder (ASD) has yet to be fully resolved. The DLD group was relatively small in the study compared to SCD and TLD groups. At an anecdotal level, it is recognised that 
UK children with DLD in elementary and high school provision are being increasingly integrated into mainstream services for general classroom support, with the result that they are unlikely to be on the caseload of a speech and language therapist and difficult to recruit to research studies. The need for increased understanding and recognition of DLD as proposed by the Catalise project (Bishop et al 2016) is acute within this context.

This was an exploratory study that relied on a novel task of metapragmatics. The fragmented nature of normative MPA research entailed difficulty in developing a clinical assessment. The task requires significant concentration skills and advanced vocabulary in the form of emotion and abstract language to describe social attributes and motivations. Distinction in group performances and more information about individual abilities might have been improved by finer measurement, including recording the number of prompts required to respond and the time taken to reply to questions as employed by Kaland, Mortensen \& Smith (2011) in a study of social communication impairment in children with Asperger Syndrome. Since this research was completed, a similar study method has been reported. Lönnqvist et al (2016) investigated the relationship between watching and interpreting aspects of pragmatics in videoed interactions for adults with high functioning autism. Using a combination of eye tracking and verbal questions during the task, Lönnqvist et al found that adults with ASD had more difficult in interpreting the videos and found differences in eye movements during the video watching. That is, adults with ASD had difficulty interpreting the pragmatic content of the videos and attending to social cues. Future research with children could include measures of eye-tracking in 
conjunction with metapragmatic testing across populations of children with SCD and DLD.

Theories of pragmatic explicitation as representational redescription were used to develop the AMP task and to interpret the findings. New directions in theory of cognitive pragmatics should continue to influence experimental studies that include questions about the nature of communication disorders. For example, Jary (2013) has proposed that the derivation of meaning from utterances can be divided into two types of implicatures (the implied meaning of the speaker): those that can be derived from the context without interpretation of the motivation of the interlocutors (material implicatures) and those in which it is necessary to understand the belief or intention of the interlocutors (behavioral implicatures). In any communicative interaction, there is skilled use of both types of meaning derivation. It should be possible in future research to test such theoretical models with children with different types of language disorder and social communication difficulties. Kissine (2016) has hypothesised, for example, that individuals with social communication impairments and autism may find it easier to process material implicatures then behavioral implicatures.

The AMP task was able to elicit pragmatic knowledge in the form of descriptions and evaluations of interloctuors' talk. Explicitation coding was feasible and has potential as an assessment of the level of children's pragmatic interpretation and social attribution. Additional research should further address the congruity between children's' pragmatic knowledge and their pragmatic performance. Preliminary work (Lockton, Collins \& Adams, 2016) found that some children with SCD have awareness of the pragmatic rules they themselves violate in conversation. Lockton et al 
recommended that the emphasis on pragmatic rule production in therapy should be replaced by intervention aimed at improving self-monitoring and social motivation. The variability in explicitation ability within the two clinical groups in this study has direct implications for management of language impairments. A thorough exploration of metapragmatic ability should influence the content and aims of intervention in terms of emphasis on development of pragmatic knowledge or selfmonitoring of rule use. New initiatives in clinical practice propose an enhanced and more active role for the child in assessment of his/her own pragmatic skills. For example, Timler's Conversation Participation Rating Scale (Timler, Boone \& Bergman 2014) includes elicitation of the child's own perceptions about how he or she is participating in a conversation as it is happening. The evolution of pragmatic assessment and intervention continues, with a renewed emphasis on functional gains in communication skills. In this area of practice, knowledge about the nature of metapragmatic skills and deficits in children with SCD or DLD could be considered central to planning targeted therapy and generalization strategies.

\section{Conclusions}

Children with Social Communication and children with Developmental Language Disorders show limited ability in reflecting on observed pragmatics as compared with children with typical language development. Contrary to predictions, metapragmatic ability in Children with SCD did not differ from that of children with DLD, neither did the groups differ on the quality of explicitation shown. Metapragmatic ability was partially dependent on structural language ability. However, since both clinical groups showed some examples of complete unawareness of pragmatic behaviours in 
interactions, this may indicate that recognition and processing of pragmatic information is restricted in many children with communication disorders. Children with SCD were less able to make social attributions to characters, once age and language were controlled for, suggesting that there is an additional social factor on top of structural language limitations in their interpretations of social interactions. Wide variations in metapragmatic ability and expression of social understanding were found across both clinical groups. Further exploration of metapragmatic ability and its role in providing a basis for self-monitoring in intervention is warranted.

\section{References}

Adams, C. (2001) Clinical diagnostic studies of children with semantic-pragmatic language disorder. International Journal of Language and Communication Disorders, 36, 289-306.

Adams, C. (2013) Pragmatic Language Impairment. In F.R Volkmar (Ed.) Encyclopedia of Autism Spectrum Disorder (pp 2320-2325). New York: Springer.

Adams, C., Cooke, R., Crutchley, A., Hesketh, A., \& Reeves, D. (2001). ACE (6-11): Assessment of Comprehension and Expression (6-11). Windsor: NFER-Nelson. Adams, C., Lloyd, J., Aldred, C.R., \& Baxendale, J. (2006) Exploring the effects of communication intervention for developmental pragmatic language impairments: a signal-generation study. International Journal of Language and Communication Disorders, 41, 41-66.

Adams, C \& Gaile, J. (2015) Social Communication Intervention Programme: Managing children's pragmatic and social communication needs in the early school years. Napier Hill Press: Cheshire. 
Adams, C., Gaile, J., Earl, G., Lockton, E., \& Freed J (2012) The Social Communication Intervention Programme: Implementation of a manualised communication intervention for school-aged children with pragmatic and social communication needs in a randomised controlled trial. International Journal of Language and Communication Disorders, 47, 3, 245-256.

American Psychiatric Association (2013). Diagnostic and statistical manual of mental disorder proposed (DSM V) (5th ed.) Washington, DC: American Psychiatric Association. American Speech and Hearing Association. Spoken Language Disorders. Retrieved from http://www.asha.org/Practice-Portal/Clinical-Topics on January 12th 2017. Axia, G., \& Baroni, M. R. (1985). Linguistic Politeness at Different Age Levels. Child Development, 56, 918-927.

Baroni, M. R., \& Axia, G. (1989). Children's meta-pragmatic abilities and the identification of polite and impolite requests. First Language, 9, 285-297. Becker, J. A. (1988). "I can't talk, I'm dead": Preschoolers' spontaneous metapragmatic comments. Discourse Processes, 11, 457-467.

Bernicot, J. (1991). French children's conception of requesting: The development of metapragmatic knowledge. International Journal of Behavioural Development, 14, 285304.

Bernicot, J., Laval, V., \& Chaminaud, S. (2007). Nonliteral language forms in children: In what order are they acquired in pragmatics and metapragmatics? Journal of Pragmatics, 39, 2115-2132.

Bishop, D. V. M. (2000). Pragmatic Language Impairment: a correlate of SLI, a distinct subgroup, or part of the autistic continuum? In D. V. M. Bishop \& L. Leonard (Eds.), 
Speech and Language Impairments in Children: Causes, Characteristics, Intervention and Outcome. Hove: Psychology Press.

Bishop, D.V.M. (2003a). The Children's Communication Checklist -2 (Second Edition). Hove: Harcourt Assessment.

Bishop, D. V. M. (2003b). Test for Reception of Grammar-Version 2. London: The Psychological Corporation.

Bishop, D. V. M., Chan, J., Adams, C., Hartley, J., \& Weir, F. (2000). Conversational responsiveness in specific language impairment: evidence of disproportionate pragmatic difficulty in a subset of children. Development and Psychopathology, 12, 177199.

Bishop, D. V., Snowling, M. J., Thompson, P. A., \& Greenhalgh, T. (2016). CATALISE: a multinational and multidisciplinary Delphi consensus study. Identifying language impairments in children (No. e1986v1). PeerJ Preprints.

Botting, N., \& Adams, C. (2005). Semantic and inferencing abilities in children with communication disorders. International Journal of Language \& Communication Disorders, 40(1), 49-66.

Bowers, L., Huisingh, R., \& LoGiudice, C.L. (2008) Social Language Development Test: Elementary. East Moline, IL: Linguisystems

Brinton, B. \& Fujiki, M. (2006) Improving peer interaction and learning in cooperative learning groups . In T.A Ukrainetz (Ed.) Contextualized language intervention: Scaffolding K-12 literacy achievements (pp289-318). Thinking Publications: Eau Claire, Wisconsin. 
Bryan, A. (1997). Colourful semantics. In Chiat, S., Law, J., \& Marshall, J. (Eds.), Language disorders in children and adults: Psycholinguistic approaches to therapy (pp143-161). London: Whurr.

Collins, A., Lockton, E., \& Adams, C. (2014). Metapragmatic explicitation ability in children with typical language development: Development and validation of a novel clinical assessment. Journal of Communication Disorders, 52, 31-43.

Davies, C., Andrés-Roqueta, C., \& Norbury, C. F. (2016). Referring expressions and structural language abilities in children with specific language impairment: A pragmatic tolerance account. Journal of Experimental Child Psychology, 144, 98-113.

Ebbels, S. (2007). Teaching grammar to school-aged children with specific language impairment using shape coding. Child Language Teaching and Therapy, 23(1), 67-93. Efron, B. \& Tibshirani, R.J. (1993). An introduction to the bootstrap. Monographs on Statistics and Applied Probability, 57, 45-53.

Gernsbacher, M. A., \& Pripas-Kapit, S. R. (2012). Who's missing the point? A commentary on claims that autistic persons have a specific deficit in figurative language comprehension. Metaphor and Symbol, 27(1), 93-105.

Gibson, J., Adams, C., Lockton, E., \& Green, J. (2013). Social communication disorder outside autism? A diagnostic classification approach to delineating pragmatic language impairment, high functioning autism and specific language impairment. Journal of Child Psychology and Psychiatry, 54, 1186-1197.

Gilliam, J. E., \& Miller, L. (2006). Pragmatic Language Skills Inventory. Austin, TX: Pro-Ed. Gillon, G., \& Dodd, B. (1995). The effects of training phonological, semantic, and syntactic processing skills in spoken language on reading ability. Language, Speech, and Hearing Services in Schools, 26(1), 58-68. 
Hickmann, M. (1993). The boundaries of reported speech in narrative discourse: some developmental aspects. In J. A. Lucy (Ed.), Reflexive Language: Reported Speech and Metapragmatics (pp. 63-90). Cambridge: Cambridge University Press.

Jary, M. (2013). Two types of implicature: material and behavioural. Mind and Language, 28, 638-660.

Ingersoll, B., Dvortcsak, A., Whalen, C., \& Sikora, D. (2005). The effects of a developmental, social-Pragmatic language intervention on rate of expressive language production in young children with autistic spectrum disorders. Focus on Autism and Other Developmental Disabilities, 20(4), 213-222.

Kaland, N., Mortensen, E. L., \& Smith, L. (2011). Social communication impairments in children and adolescents with Asperger syndrome: Slow response time and the impact of prompting. Research in Autism Spectrum Disorders, 5(3), 1129-1137.

Karmiloff-Smith, A. (1986). From meta-processes to conscious access: Evidence from children's metalinguistic and repair data. Cognition, 23, 95-147.

Klin, A. (2000). Attributing social meaning to ambiguous visual stimuli in higherfunctioning autism and Asperger syndrome: the social attribution task. Journal of Child Psychology and Psychiatry, 41(7), 831-846.

Kissine, M. (2015). Pragmatics as Metacognitive Control. Frontiers in Psychology, 6, 2057. http://doi.org/10.3389/fpsyg.2015.02057

Landa, R (2013) Pragmatic rating Scale. In F.R. Volkmar (Ed.) Encyclopedia of Autism Spectrum Disorders (pp 2327-2331). New York: Springer.

Landis, J. R., \& Koch, G. G. (1977). The measurement of observer agreement for categorical data. Biometrics, 33 (1), 159-174. 
Laval, V. (2003). Idiom comprehension and metapragmatic knowledge in French children. Journal of Pragmatics, 35, 723-739.

Law, J., Campbell, C., Roulstone, S., Adams, C., \& Boyle, J. (2008). Mapping practice onto theory: the speech and language practitioner's construction of receptive language impairment. International Journal of Language \& Communication Disorders, 43(3), 245263.

Lockton, E., Adams, C., \& Collins, A. (2016). Do children with social communication disorder have explicit knowledge of pragmatic rules they break? A comparison of conversational pragmatic ability and metapragmatic awareness. International Journal of Language \& Communication Disorders, 51, 508-517.

Lönnqvist, L., Loukusa, S., Hurtig, T., Mäkinen, L., Siipo, A., Väyrynen, E., ... \& Ebeling, H. (2016). How young adults with autism spectrum disorder watch and interpret pragmatically complex scenes. The Quarterly Journal of Experimental Psychology. Advanced online publication. http://dx.doi.org/10.1080/17470218.2016.1233988 Marton, K., Abramoff, B., \& Rosenzweig, S. (2005). Social cognition and language in children with specific language impairment (SLI). Journal of Communication Disorders, $38,143-162$.

Meline, T. J., \& Brackin, S. R. (1987). Language-impaired children's awareness of inadequate messages. Journal of Speech and Hearing Disorders, 52, 263-270. Merrison, S., \& Merrison, A. J. (2005). Repair in speech and language therapy interaction: Investigating pragmatic language impairment of children. Child Language Teaching and Therapy, 21(2), 191-211. 
Norbury, C. F. (2004). Factors supporting idiom comprehension in children with communication disorders. Journal of Speech, Language, and Hearing Research, 47(5), 1179-1193.

Norbury, C. F. (2014). Practitioner review: Social (pragmatic) communication disorder conceptualization, evidence and clinical implications. Journal of Child Psychology and Psychiatry, 55(3), 204-216.

Norbury, C. F., \& Bishop, D. V. (2003). Narrative skills of children with communication impairments. International Journal of Language \& Communication Disorders, 38(3), 287-313.

O'Neill, D. K. (2007). The language use inventory for young children: A parent-report measure of pragmatic language development for 18-to 47-month-old children. Journal of Speech, Language, and Hearing Research, 50(1), 214-228.

Phelps-Terasaki, D., \& Phelps-Gunn, T. (2007). TOPL-2 Test of Pragmatic Language. Austin,Tx: Pro-Ed.

Raven, J. C. (1979). Coloured Progressive Matrices. Oxford: Oxford Psychologists Press Limited.

Richardson, K., \& Klecan-Aker, J. S. (2000). Teaching pragmatics to language-learning disabled children: A treatment outcome study. Child Language Teaching and Therapy, 16(1), 23-42.

Rutter, M., Bailey, A., \& Lord, C. (2003). The Social Communication Questionnaire (SCQ). Los Angeles, CA: Western Psychological Services.

Sheng, L., \& McGregor, K. K. (2010). Lexical-semantic organization in children with specific language impairment. Journal of Speech, Language, and Hearing Research, $53,146-159$. 
Shwe, H. I., \& Markman, E. M. (1997). Young children's appreciation of the mental impact of their communicative signals. Developmental Psychology, 33(4), 630. Semel, E., Wiig, E., \& Secord, W. (2006). Clinical Evaluation of Language Fundamentals Fourth Edition (UK). London: Harcourt Assessment.

Skarakis-Doyle, E. (2002). Young Children's Detection of Violations in Familiar Stories and Emerging Comprehension Monitoring. Discourse Processes, 33(2), 175-197. Tager-Flusberg, H., Paul, R., \& Lord, C. (2005). Language and communication in autism. Handbook of autism and pervasive developmental disorders, 1, 335-364.

Timler, G. R., Olswang, L. B., \& Coggins, T. E. (2005). "Do I know what I need to do now?" A social communication intervention for children with complex clinical profiles. Language, Speech and Hearing Services in Schools, 36, 73-85.

Timler, G. R., Boone, W. J., \& Bergmann, A. A. (2014). Development of the Conversation Participation Rating Scale: Intervention planning implications for two school-age children with autism spectrum disorders. Topics in Language Disorders, 34(3), 252-267. Tomblin, J. B., Records, N. L., Buckwalter, P., Zhang, X., Smith, E., \& O'Brien, M. (1997). Prevalence of specific language impairment in kindergarten children. Journal of Speech Language and Hearing Research, 40, 1245-1260.

Tsuji, H., \& Doherty, M. J. (2014). Early development of metalinguistic awareness in Japanese: Evidence from pragmatic and phonological aspects of language. First Language, 34(3), 273-290.

Van Kleeck, A., Gillam, R. B., \& McFadden, T. U. (1998). A study of classroom-based phonological awareness training for preschoolers with speech and/or language disorders. American Journal of Speech-Language Pathology, 7(3), 65-76. 
Wilkinson, L. C., \& Milosky, L. M. (1987). School age children's metapragmatic knowledge of requests and responses in the classroom. Topics in Language Disorders, 7, $61-70$

Wilson, D., \& Sperber, D. (2015). Outline of relevance theory. HERMES-Journal of Language and Communication in Business, 3(5), 35-56.

Yifat, R., \& Zadunaisky-Ehrlich, S. (2008). Metapragmatic comments indexing conversational practices of preschool children in institutional discourse. First Language, $28,329-347$.

Zwitserlood, R., Wijnen, F., Weerdenburg, M., \& Verhoeven, L. (2015). 'MetaTaal': enhancing complex syntax in children with specific language impairment-a metalinguistic and multimodal approach. International Journal of Language \& Communication Disorders, 50(3), 273-297.

\section{Acknowledgments}

We acknowledge with gratitude the time and efforts of all participants and referring speech and language practitioners for their support. Thanks also to Jenny Freed, Jenny Gibson and Jacqueline Gaile for extra testing and to Graham Dunn for statistical support. The original research was supported by an Economic and Social Research Council Grant to Adams and Lockton (reference number: RES-000-222223).

\section{Ethics}


The study design was approved by the UK NHS Northern and Yorkshire Multi-Centre Research Ethics Committee (07/MRE03/3). Parents were provided with written information about the study and provided signed consent on behalf of their children. Informed consent was also gained from the child (where able). 